\title{
SUPPRESSION DU MASSAGE E'T DU LAVAGE DE LA MAMELLE CHEZ LES VACHES LAITIERES EFFETS SUR LES CARACTÉRISTIQUES DE TRAITE ET SUR LA QUALITÉ BACTÉRIOLOGIQUE DU LAIT
}

\author{
J. LABISSIËRE, J. RICHARI)*, J. F. COMHALD \\ et $F$. A. IE LA CHEVAJERIE: \\ Laboratoive de la Traite, \\ Centre de Recherches de Rennes, I.N.R.A., \\ 65, rue de Saint-Brieuc, 35042 Rennes \\ * Laboratoire de Technologie laitiire. \\ Centre national de Recherches zootechniques, I. N.R. A., \\ 78350 Jouy-en-Josas
}

\begin{abstract}
RÉSUMÉ
1. Trente-trois primipares françaises Frisonne Pie Noire sont réparties, au fur et à mesure de leur mise bas, alternativement dans deux lots :

a) Pour le lot témoin (i 6 vaches), la mamelle est massée penclant zo secondes arec un lingc humide préalablement trempé dans de l'eau à $45^{\circ} \mathrm{C}$. I a pose des gobelets suit immédiatement ce nettoyage.

b) Pour le lot essai ( $\mathrm{r} 7$ vaches), cettc préparation est totalement suppriméc depuis le vêlage jusqu'au tarissement.

2. Dans ce dernicr cas :

a) La production du lait en 300 jours est réduite de 2,3 I p. ioo alors que celle des matières grasses et des matières azotées est respectivement accrue de 5,82 p. Ioo et 2,6 I p. Ioo. Aucune de ces 3 différences n'est significative. Le taux butyreux et le taux azoté se révèlent par contre statistiquement plus élevés et le volume de lait recueilli à l'égonttage est en moyenne supérieur de $100 \mathrm{ml}$ par traite.

b) Bien que le délai séparant la pose des gobelets ct l'arrivée du lait alvécolaire soit toujours plus long en l'absence de nettoyage on ne constate pas pour autant d'allongement sur le temps d'écoulement du lait. Ceci résulte probablement du fait que le débit maximum cst alors nettement plus important.

c) Bien que restant en dessous des limites généralement considérécs comme incliquant une mammite subclinique la notation C.M.T. est plus élevée chez les vaches ne recevant aucune préparation.

d) La contamination microbienne du lait provenant des mamelles non lavées demeure en moyenne inférieure aux 100000 germes totaux/ml qui correspond aux normes admises actucllement par la législation française pour le paiement du lait en qualité A. Si les mamelles sont propres au moment de la traite, grâce, par exemple, à des conditions d'élevage adéquates, la contamination du lait qui résulterait de l'absence de lavage peut être négligée.
\end{abstract}




\section{I. - INTRODUCTION}

On entend généralennent par préparation à la traite l'ensemble des manipulations qui consistent, avant la pose des gobelets, à laver la mamelle avec un linge humide et chaud (ou avec une douchette) et à extraire quelques jets de lait de chacun des trayons.

Cette opération a d'abord été recommandée dans un but hygiénique puisqu'en réduisant la quantité d'impuretés introduites dans le lait elle améliore la qualité bactériologique du produit récolté.

Mais, il est également bien admis maintenant (J. LABussière et Ph. Richard, I965 et R. DENAMUR, I965) que ce "massage du pis ", généralement vigoureux, constitue l'un des meilleurs "stimuli " pour déclencher le réflexe neuroendrocrinien d'éjection décrit par Eily et PeTERSEN en I94I.

Il importe toutefois de souligner que pour être pleinement efficace cette stimulation doit être effectuée en tenant compte d'un certain nombre de précautions qui risquent de devenir difficiles à respecter lorsque trop de vaches sont traites simultanément par une seule personne. C'est ainsi, par exemple, que la sensibilité au massage varie d'un animal à l'autre et PhILI,IPS (I965) a démontré qu'il est nécessaire d'appliquer à chacun le temps de lavage qui lui convient si l'on souhaite extérioriser complètement ses potentialités laitières.

C'est ainsi également que le temps qui sépare la fin de la préparation et la pose des gobelets doit être le plus court possible puisque l'ocytocine est détruite rapidement après sa libération (GiNsburg et Sinth, I9.59; Fitziratrick, ig6I). Nous avons pu démontrer que la production de lait est réduite de $5,3 \mathrm{p}$. Ioo, I5,4 p. Ioo et 29,7 p. Ioo lorsque le délai entre le lavage des manelles et la pose des gobelets est respectivement de 2,6 et I 3 minutes (LABUssière, I970), et dans ce cas également, les animaux réagissent très différemment à une attente prolongée ( ${ }^{1}$ ).

Dans les grandes exploitations, il est difficile de bien connaître les exigences individuelles des animaux et la pose inmédiate des gobelets n'est pas toujours conciliable avec l'organisation du travail dans les salles de traite en épi qui sont très répandues. Il nous est done apparu important de savoir s'il n'est pas préférable dans de tels cas de supprimer complètement la préparation de la mamelle ce qui offrirait l'avantage de simplifier le travail du trayeur et d'augmenter sa productivité horaire.

Un certain nombre de résultats contradictoires ont déjà été publiés à ce sujet par Knoop et Monroe (I950), Whittrlestone, Parkinson et McFetridge (I957), Philitips (ig60), Murray et Lorna G. Lightbody (ig62), Wilson (ig63), Ipsen (i964), Philitips, Cowhig et Walshe (i967), Griffin (ig68), Momongan et Schmidt (I970), Morag, Saran-Rosenzuaig et Sagi (I973), Barowicz et Styczynsky (I974).

Les divergences relevées à la lecture de ces travaux traduisent probablement le fait que les essais portent généralement sur un nombre limité d'animaux et sur des périodes expérimentales relativement courtes ne permettant pas de tenir compte des effets cumulatifs néfastes qui pourraient résulter de rétentions répétées dans les acini mammaires.

(1) Il nous semble justifić d'ćtablir à ce sujet un parallélisme entre ces variations de comportement et l'aspect très hétérogene des courbes de pression intramammaires consécutives à un massage. 
Les recherches que nous avons effectuées en I 969 et I970 et que nous rapportons ci-après couvrent par contre une lactation entière ; elles présentent également l'intérêt d'être réalisées sur des génisses qui n'ont jamais été soumises au préalable à une technique de traite différente de celle dont nous souhaitions tester l'efficacité.

\section{II. - MATÉRIEL E'T MÉTHODE}

\section{A. - - Technique de traite du lot témoin et du lot essai}

Trente-trois génisses françaises Frisonnes Pie Noire (FFPN) ayant vêlé entre le I 5 janvier I 969 et le I9 novembre I 969 sont réparties, au fur et à mesure de leur mise bas, alternativenent dans 2 lots qui reçoivent chaque jour pendant toute la lactation les traitements suivants :

\section{Lot témoin $T$ (16 animanx).}

La mamelle est massée pendant 30 secondes avec un linge humide préalablement trempé dans de l'eau à $45^{\circ} \mathrm{C}$ puis essoré. La pose des gobelets suit immédiatement cette préparation.

L'aliment concentré complétant la ration de base est distribué individuellement à l'étable, après la traite ${ }^{1}$ ). Le réajustement de la ration à la production laitière de chaque vache est effectué tous les $I_{5}$ jours.

\section{Lot essai E (17 animaux).}

Le nettoyage du pis (qui précède la pose des gobelets) est totalenent supprimé (lepuis le vêlage jusqu'au tarissement.

Il importe de préciscr à ce sujet que : diverses :

- les mamelles n'ont jamais été rasées ce qui peut favoriser l'accrochage des impuretés

- les vaches sont logées en stabulation courte entravée oì la paille est changée quotidiennement à l'exception du dimanche.

Contrairement au lot T, l'aliment concentré est distribué en salle cle traite, juste après l'entrée de l'animal dans sa stalle $\left({ }^{2}\right)$.

lin dehors des différences signalées ci-dessus, les 2 groupes sont traits dans des conditions identiques : la dépose des gobelets est précédée par un égouttage à la machine bref, dont la durée est laissée à l'appréciation du traycur; la traite est assurée 2 fois par jour ( 6 h 30 ct 16 h I5) à l'aide d'une machine à traire Alfa-Laval dont les principales caractéristiques sont les suivantes : niveau de vide : $33 \mathrm{~cm} / \mathrm{Hg}$; 60 pulsations $/ \mathrm{mn}$; rapport succion-massage : 70 p. Ioo ; manchons : $n^{\circ} 960-0020$ et gobelets : $n^{\circ} 25654$.

Ia salle de traite est du type parallèle à 3 postes.

\section{B. - Contrôles des résultats}

\section{r. Production et composition du lait.}

Le volume de lait produit par chaque vache est noté à chaque traite avec une précision voisine de roo $\mathrm{ml}$.

Un échantillon destiné à l'analyse de la matière grasse (méthode Gerber) et de lá matière azotée (méthode du Noir Amido) est prélevé, matin et soir, tous les 28 jours, à partir de la $3^{\text {e }}$ semaine de lactation.

Toutes les productions sont rapportées à une lactation de zoo jours à l'aide de la méthode Fleishman.

(') Ration cle base :

- ro $\mathrm{kg}$ de foin de pré,

- $20 \mathrm{~kg}$ d'ensilage d'herbe de prairie permanente auquel est incorporé ${ }_{50} g$ de condiment ninéral.

(2) Comme dans le cas précédent, les rations sont recalculées tous les ${ }_{5} 5$ jours cri fonction du niveau individuel de sécrétion. 
2. Fitat sanitaive de la manclle.

Toutes les + semaines, l'état sanitaire de chaque quarticr est apprécié à l'aide de l'épreuve CMT (California Mastitis Test).

Le lait est prélevé environ 3 heures avant la traite du soir.

\section{Cinétique d'émission du lait.}

Le rolume de lait de chaque quartier est mesuré toutes les 6 secondes avec une précision supéricure à $5 \mathrm{ml}$ grâce à une chaîne d'enregistrement (LABUssière et MARTINET, I964) comprenant + suiveurs de niveaux SEIRAM (SN 50 ) un commutateur de voies, un voltmètre digital MACQ et une imprimante à boule IBM.

Ces contrôles sont effectués à la traite du soir pendant les 2 premières semaines qui suivent le vêlage puis toutes les 4 semaines pendant 4 jours consécutifs.

\section{Qualité bactériologique du lait.}

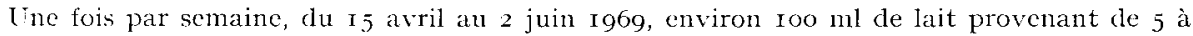
6 vaches de chaque lot sont prélevés dans les bocaux de mesure, après une homogénéisation. Les échantillons sont regroupés pour constituer un échantillon unique d'environ I/2 1 pour chaque lot sur lequel sont effectués les dénombrements microbiens suivants à $30^{\circ} \mathrm{C}$

- "flore thermorésistante " même méthocle de numération que pour la flore totale, le lait étant préalablement chauffé 30 minutes à $63,5^{\circ} \mathrm{C}$,

- spores aérobies-mésophiles, suivant la méthode du CNERNA (I962),

- Clostridium fermentant le lactate, sur milieu de BRYANT et BUURKey (I956),

- bactéries coliformes sur milicu RVB (Difco no ${ }^{\circ} 2$ ) après incubation des boîtes cle l’etri I 8 à 24 heures a $30^{\circ} \mathrm{C}$.

Iin outre, la filtration de 0,5 litre de lait sur un disque d'ouate permet d'attribuer une note de propreté à chaque échantillon en fonction d'une échelle inspirée de celle proposéc par ScHNEIDER (1954) ct reproduite à la figure $I$.

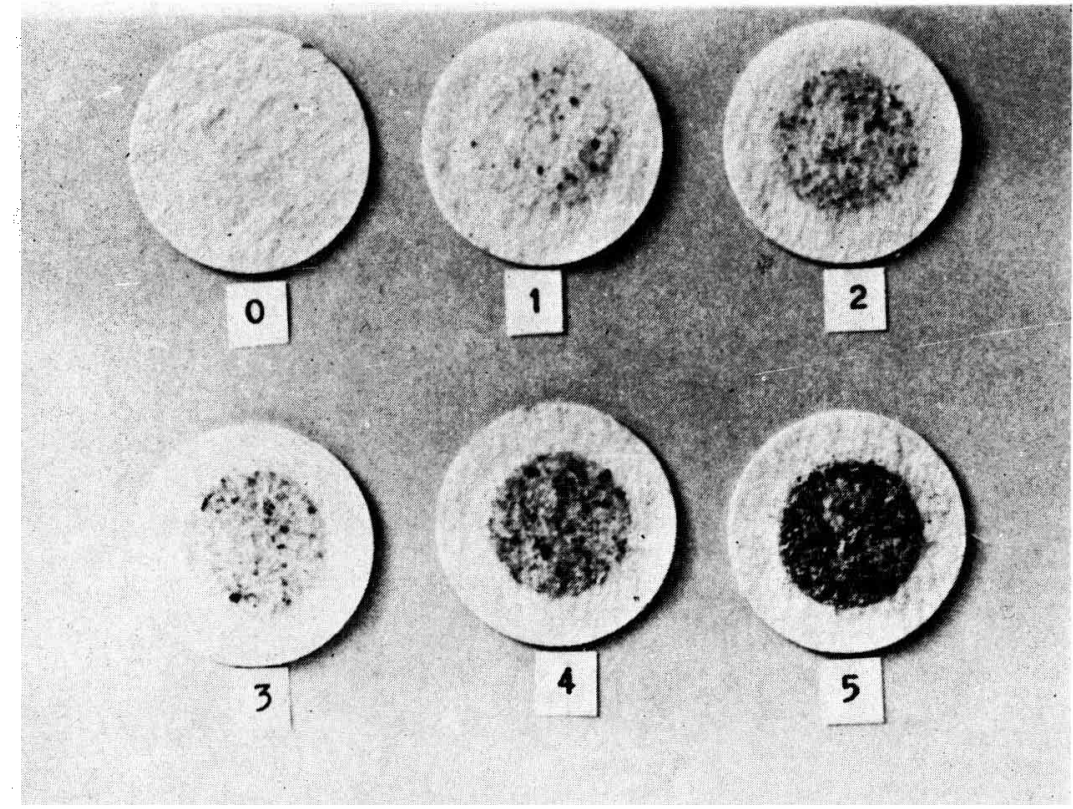

FIG. I. - Notation de l'épreuve de filtration stur outate (d'après C. Schneider) 
Le jour du prélèvement, la contamination du lait par la machine à traire est réduite au maximum grâce à un démontage des parties les plus compliquées (vannes et joints) et à un nettoyage du matériel comportant la désinfection des faisceaux trayeurs à la vapeur et celle du reste de l'installation avec unc solution d'iodophores à $50 \mathrm{mg} / \mathrm{l} \mathrm{d}$ 'iode.

5. Expérience $n^{\circ} 2$ (complémentaire).

Les aires paillées de notre stabulation entravée en stalle courte étant renouvelées quotidiennement il nous a paru intéressant de réaliser un essai complémentaire sur un autre troupeau vivant en stabulation libre.

Au moment de la traite, 52 vaches sont réparties en 2 lots de 26 selon l'état de propreté apparente des mamelles (tabl. 2). Dans chaque lot, la moitié des pis sont lavés et l'autre non. Les faisceaux trayeurs sont désinfectés à la vapeur.

Une dérivation montée sur le tıyau à lait permet de recueillir environ 0,5 litrs par animal afin d'effectuer les mêmes examens bactériologiques que dans l'expérience précédente.

\section{III. - RÉSULTATS}

\section{A. - Production et composition du lait}

La figure 2 présente pour chaque lot, l'évolution au cours de la lactation, de la production du lait (fig. $2 a$ ) et de ses principaux constituants (fig. $2 b$ et $2 d$ ). Il apparaît à l'examen de ces courbes que la sécrétion de la mamelle est peu affectée par la suppression du massage à l'eau chaude qui précède la pose des gobelets.

L'analyse statistique (tabl. I) ne révèle d'ailleurs aucune différence entre lots pour les quantités de lait, de matières grasses et de matières azotées recueillies en 300 jours (respectivement : 2,3 p. Ioo ; $+5,8 \mathrm{p}$. Ioo et $+2,6 \mathrm{p}$. IOo). Il importe de remarquer que le gain constaté dans le lot essai pour ces deux derniers critères résulte d'une augmentation significative du taux butyreux et du taux azoté (tabl. I, fig. $2 c$ et $2 e)$.

\section{B. - Cinétique d'émission du lait au cours de la traite}

Le volume de " lait machine » est peu modifié par la suppression du massage de la mamelle (fig. $3 a$ ), mais la fraction recueillie à l'égouttage est par contre sensiblement plus élevée (environ Ioo $\mathrm{ml}$ : fig. 3 b). Le délai séparant la pose des gobelets et l'arrivée du lait alvéolaire dans le récipient de contrôle est toujours plus long en l'absence de nettoyage (fig. $3 \mathrm{c}$ ). Il est probable que le réflexe d'éjection est alors induit par les mouvements rythmiques des manchons trayeurs et il importe de remarquer que ce phénomène est déjà observé au cours des premiers jours de la lactation (fig. $3^{d}$ ). Cette évacuation différée du lait alvéolaire ne se traduit pas, pour autant, par un allongement du temps d'écoulement du lait qui est sensiblement identique dans les 2 lots (fig. $3 e$ ). Ceci résulte probablement du fait que le débit maximum est nettement plus important chez les animaux ne recevant aucune préparation (fig. $3 f$ ) que chez ceux du lot témoin.

\section{C. - Etat sanitaire de la mamelle}

La notation CMT est plus élevée chez les vaches dont la mamelle n'est pas " massée " (fig. 4). Elle reste néanmoins toujours inférieure à I (soit moins de 400 ooo à 500 ooo cellules par millilitre), c'est-à-dire en dessous des limites généralement considérées comme indiquant une mammite subclinique. 

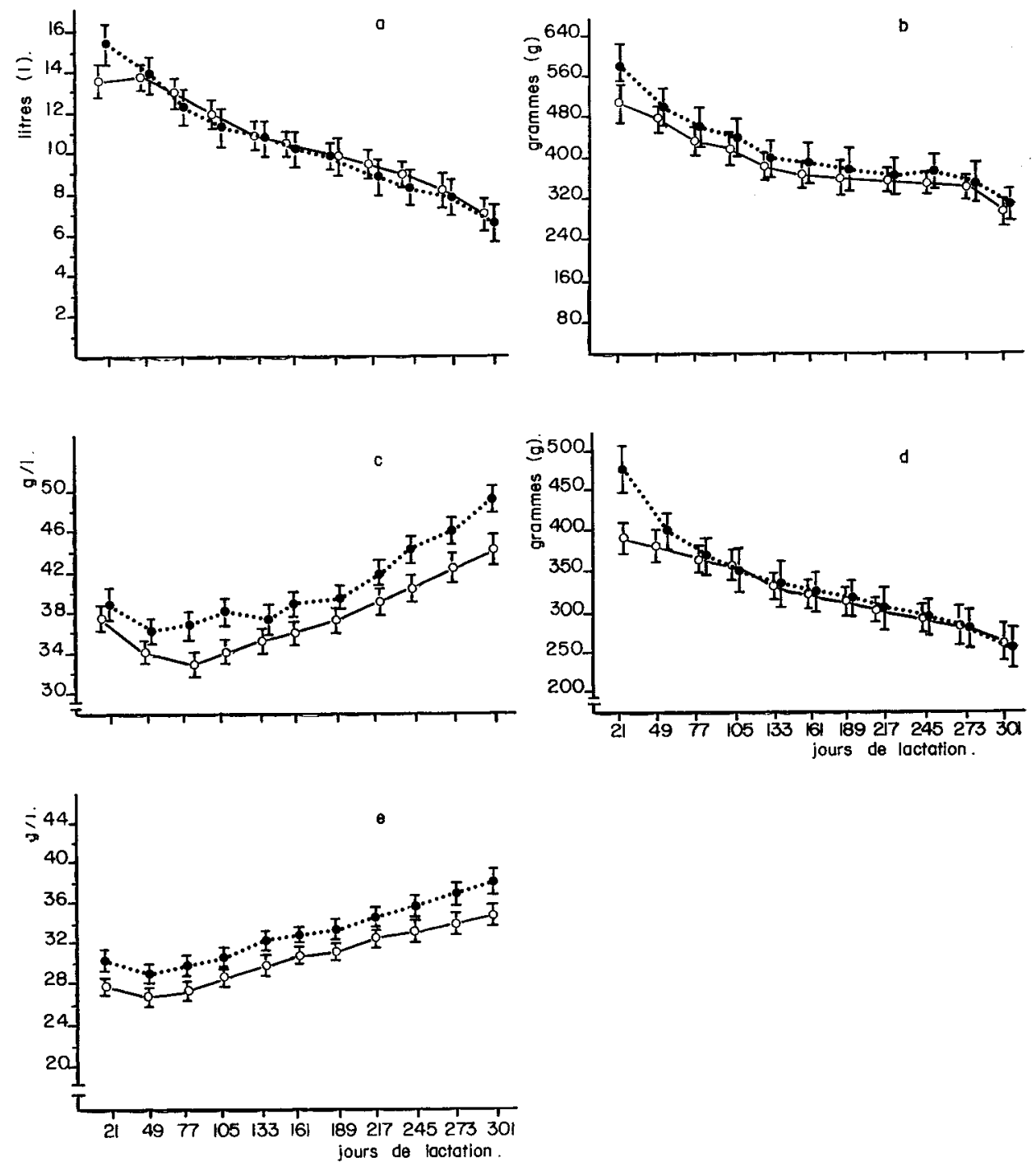

Irig. 2. - Evolution de la production du lait et de ses principant constituants au cotr's de la lactation lot témoin $-0-0-0$
lot essai $\ldots \ldots$

a) production laitière

b) production de matieres grasses totales,

c) taux butyreux,

d) production de matières azotécs totales,

e) taux azoté.

Les valeurs présentées correspondant aux moyennes journalières de chaque lot assorties des erreurs type de la moyenne. 

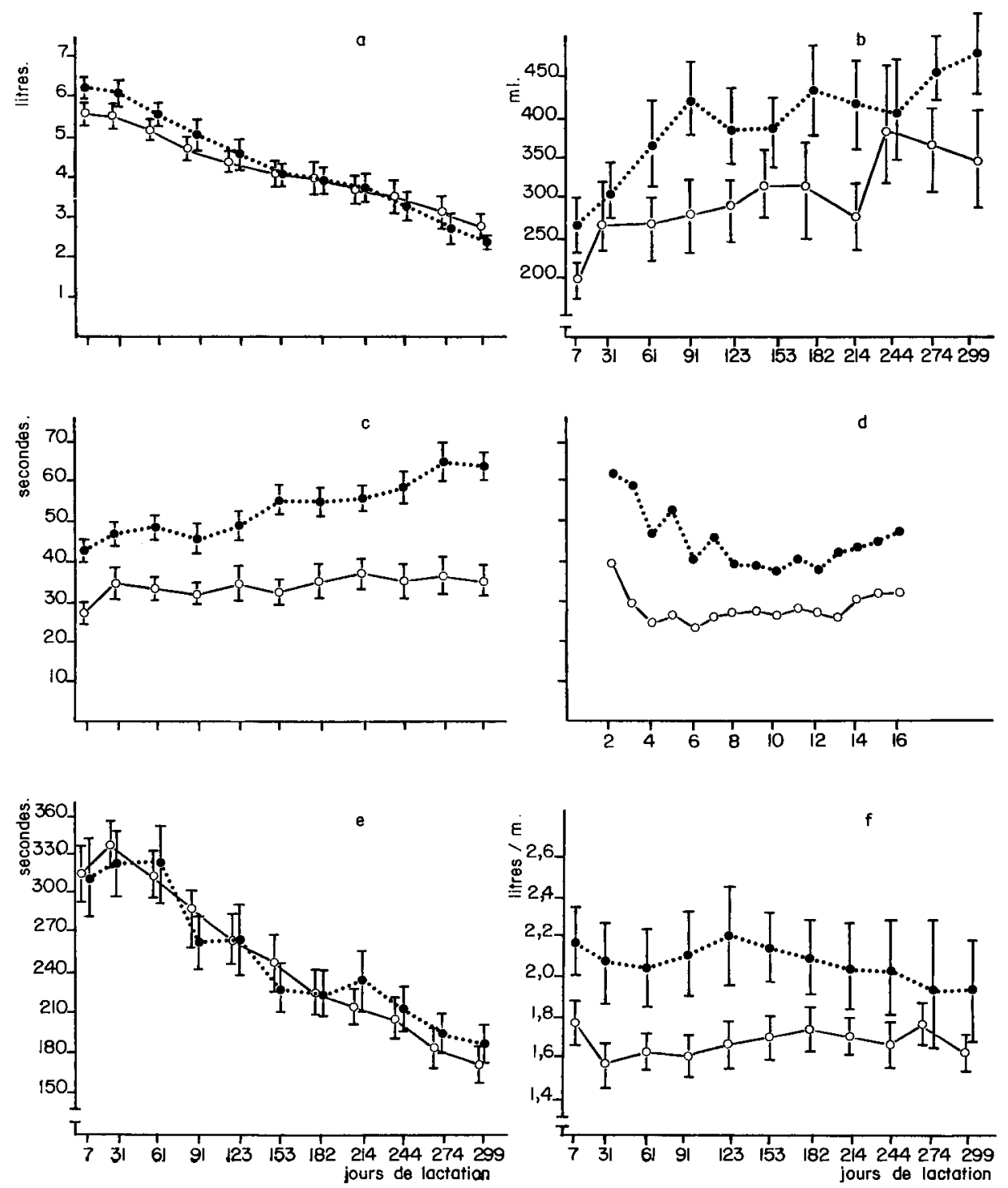

FiG. 3. - Erolution de quelques paramètyes caractérisant

la cinétique d'émission du lait au coutrs de la traite du soir

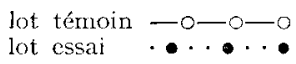

a) volume de lait obtenu à la machine,

b) volume de lait recueilli à l'égouttage,

c) délai entre la pose des gobelets et la sortie du lait alvéolaire (lactation complètc),

d) délai entre la pose des gobelets et la sortie du lait alvéolaire (i 6 preniers jours),

e) temps d'écoulement du lait.

f) débit maximum.

Les valeurs présentées correspondent aux moyennes de chaque lot (traite du soir) assorties des erreurs type de la moyenne. 
TABLEAU I

Effets de la suppression de la préparation de la mamelle sur la production du lait et sur celle de ses principaux constituants

\begin{tabular}{|c|c|c|c|c|c|}
\hline & \multicolumn{2}{|c|}{$\begin{array}{l}\text { Lot témoin ( } \mathrm{T}) \\
\text { avec massage } \\
\text { (16 primipares) }\end{array}$} & \multicolumn{2}{|c|}{$\begin{array}{l}\text { Lot essai (E) } \\
\text { sans massage } \\
\text { (17 primipares) }\end{array}$} & \multirow[t]{2}{*}{$\begin{array}{c}\text { Test } t \\
31^{\circ} \text { de liberté }\end{array}$} \\
\hline . & moyenne & écart-type & moyenne & écart-type & \\
\hline Production laitière (i en $300 \mathrm{j}$ ).... & 3301,11 & 623,77 & 3) 225,17 & 926,46 & 0,275 \\
\hline Production de MG (kg en $300 \mathrm{j}$ ) . & 120,10 & $2.2,03$ & 127,42 & $40,4 t^{\prime}$ & 0,613 \\
\hline Production de MA (kg en $300 \mathrm{j}$ ) ... & 99,43 & 16,75 & $10,0,03$ & 28,60 & 0,316 \\
\hline Taux butyreux journalier moyen & & & & & \\
\hline$(g / \mathrm{I}) \ldots \ldots \ldots \ldots \ldots \ldots \ldots \ldots$ & 36,59 & 3,17 & $39,1^{\prime}$ & 3,60 & $2,157\left({ }^{1}\right)$ \\
\hline Taux azoté journalier moyen $(g / 1)$. & $30, \geq 3$ & 1,37 & $31,8^{\prime}$ & 1,80 & $2,867\left({ }^{2}\right)$ \\
\hline
\end{tabular}

( $\left.{ }^{1}\right)$ Significatif au seuil $0,05(t>2,0 \div 2)$.

(2) Significatif au seuil $0,01(t>2,750)$.

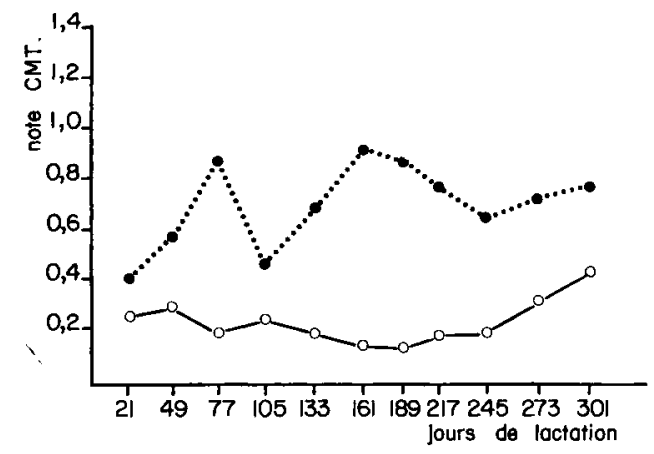

FIG. 4. - Evaluation sommaire du nombre de cellules dans le lait an cours de la lactation

Test CMT

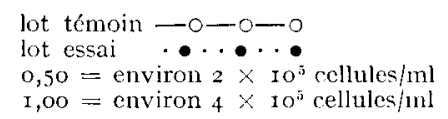

Les valeurs présentées correspondent à la moyenne de chaque lot

Il importe de souligner que certaines vaches soumises à la suppression de la préparation, et présentant dès le vêlage des réactions CMT positives sur certains quartiers n'ont jamais évolué vers une mammite du type aigu. 


\section{D. - Qualité bactériologique du lait}

Les résultats des examens bactériologiques effectués sur le lait de nos 2 expériences figurent dans le tableau 2. A titre de comparaison et pour faciliter la discussion, nous avons reproduit également dans ce tableau les résultats d'essais réalisés par Cousins (I972).

\section{TABLEAU 2}

Qualité bactériologique du lait produit en fonction de l'état de propreté des mamelles (Moyenne arithmétique du nombre de microorganismes $/ \mathrm{ml}$ )

\begin{tabular}{|c|c|c|c|c|c|c|c|}
\hline $\begin{array}{l}\text { Expérience } \\
\text { et auteurs }\end{array}$ & Mamelles & $\begin{array}{l}\text { Note } \\
\text { de pro- } \\
\text { preté }\end{array}$ & $\begin{array}{l}\text { Flore } \\
\text { totale }\end{array}$ & $\begin{array}{c}\text { Bactéries } \\
\text { coli- } \\
\text { formes }\end{array}$ & $\begin{array}{c}\text { Flore } \\
\text { thermo- } \\
\text { résistante }\end{array}$ & $\begin{array}{l}\text { Sporulés } \\
\text { aérobies } \\
\text { mésophiles }\end{array}$ & $\begin{array}{l}\text { Nombre } \\
\text { d'exa- } \\
\text { mens }\end{array}$ \\
\hline $\begin{array}{c}\text { Expérience } n^{\circ} 1 \\
\text { (principale) }\end{array}$ & $\begin{array}{l}\text { Assez propres : } \\
\quad \text { - non lavées ... } \\
\text { - lavées ...... }\end{array}$ & $\begin{array}{l}2,7 \\
1,5\end{array}$ & $\begin{array}{l}33000 \\
10000\end{array}$ & $\left.\begin{array}{l}44 \\
24\end{array}\right\}$ & $\begin{array}{l}65 \\
21\end{array}$ & $\begin{array}{r}36 \\
8\end{array}$ & $\begin{array}{l}8^{a} \\
8^{a}\end{array}$ \\
\hline $\begin{array}{l}\text { Expérience no } 2 \\
\text { (complémentaire) }\end{array}$ & $\begin{array}{l}\text { Sales non lavées ... } \\
\text { Propres non lavées } \\
\text { Sales lavées ..... } \\
\text { Propres lavées .... }\end{array}$ & $\left.\begin{array}{l}3,5 \\
2,2 \\
1,1 \\
1,1\end{array}\right\}$ & $\begin{array}{r}130000 \\
7100 \\
4600 \\
3700\end{array}$ & $\left.\begin{array}{r}57 \\
6 \\
1 \\
2\end{array}\right\}$ & $\left.\begin{array}{r}170 \\
110 \\
14 \\
13\end{array}\right\}$ & $\left.\begin{array}{r}72 \\
38 \\
5 \\
5\end{array}\right\}$ & $\begin{array}{l}13^{b} \\
13^{b} \\
13^{b} \\
13^{b}\end{array}$ \\
\hline $\begin{array}{c}\text { Expérience de } \\
\text { (Cousins, 1972) }\end{array}$ & $\begin{array}{r}\text { Non lavées }(\mathbf{1}) \ldots . \\
\text { Lavées (eau pure }\left(^{2}\right) \\
\text { ou javellisée) .... }\end{array}$ & $一$ & $\begin{array}{r}100(1) \\
300(1)\end{array}$ & $\begin{array}{l}1\left({ }^{1}\right) \\
1\left(^{2}\right)\end{array}$ & $\begin{array}{r}160\left(^{1}\right) \\
3\left(^{2}\right)\end{array}$ & $\begin{array}{l}23\left({ }^{(1)}\right. \\
1\left(^{2}\right)\end{array}$ & $\begin{array}{l}18 \\
12\end{array}$ \\
\hline
\end{tabular}

a Examen sur lait de mélange de 5 à 6 vaches.

$b$ Examen sur lait de vaches individuelles.

( $\left.{ }^{1}\right)$ Résultats moyens de 3 expériences faites sur 6 vaches chacune.

(2) Résultats moyens de 2 expériences faites sur 6 vaches chacune.

Les moyennes réunies par une accolade (exp. 1 et 2) ne diffèrent pas significativement au setril $P=0,05$ ).

Comme on pouvait s'y attendre, la qualité bactériologique du lait est bien meilleure lorsque les mamelles sont lavées avant la traite. On observe cependant des différences appréciables de qualité bactériologique d'une expérience à l'autre non seulement en 1'absence de nettoyage - ce qui peut être expliqué par des différences de propreté des pis - mais aussi après le lavage de ceux-ci. Dans ce cas, les différences de qualité peuvent être attribuées à un nettoyage imparfait de la glande s'ajoutant dans l'expérience I à une contamination possible du lait par le matériel de traite relativement compliqué. Lorsque les mamelles visiblement sales (maculées de bouse) ne sont pas lavées (expérience $\mathbf{n}^{\circ} 2$ ), le nombre de germes totaux dépasse en moyenne I00 $000 / \mathrm{ml}$ de lait mais le nombre de bactéries coliformes reste inférieur à $\mathrm{ro0} / \mathrm{ml}$. Les résultats sont en bon accord avec ceux obtenus par C. K. JoHns (I962) et montrent qu'une contamination du lait par des impuretés d'origine fécale n'augmente pas considérablement le nombre de germes totaux ou de bactéries coliformes dans le lait. 
Le lait obtent dans l'expérience I contenait moins de 0,2 spore/m1 de Clostridium fermentant le lactate (que les mamelles soient lavées ou non) mais ceci est peut-être dû au fait que l'ensilage consommé par les vaches était d'excellente qualité.

\section{IV. - DISCUSSION}

La suppression du massage et du nettoyage du pis ne semble pas affecter d'une façon sensible l'activité sécrétoire de la mamelle.

Nous n'avons en effet relevé aucune incidence significative, ni sur la production du lait, ni sur celle de matières grasses ou de matières azotées; nos résultats confirment en cela ceux de Knoop et Monroe (I950), Murray et Lorna. G. Lightbody (ig62), IpSen (I964), Griffin (Ig68), Momongar et Schmidt (ig70), Morag, SaranRosenzUaig et SAgI (I973), BARowicz et STYCYZYNSKy (I974) puisque pour tous ces auteurs l'écart entre lots témoin et expérinental pour ces différents critères de production n'excède jamais 2 p. Ioo.

Il est toutefois prudent d'éviter une généralisation hâtive et de tenir compte en particulier :

a) De la race qui semble jouer un rôle important. C'est ainsi qu'en NouvelleZélande, sur des vaches Jerseyaises, Whi'tritestone, Parkinson et Mac Frtridge (I957) constatent une réduction de I6 p. Ioo du rendement en lait et que PHII,LIPS (I960) signale pour les matières grasses une perte de $32 \mathrm{p}$. Ioo qu'il n'a pu confirmer quelques années plus tard sur des vaches Frisonnes $(-8,4$ p. Ioo non significatif; Phil,itis, Cowhig et Walshi, ig67).

b) De la qualité et de la durée de la préparation puisque les travaux de WirsoN (I963) démontrent que la perte consécutive à la suppression complète de cette opération (- I I p. IOo) est inférieure à celle occasionnée par un massage trop rapide (- 23,8 p. IOO).

L'obtention presque normale dı lait alvéolaire en l'absence de massage ne doit pas surprendre si l'on sait que :

I. Des quantités importantes d'ocytocine sont dosées dans le plasma de vaches n'ayant reçu aucune préparation (Momongan et Schmid's, I970; Barowicz et STYCZYNSKY, 1974).

2. Des sollicitations d'origine extramammaire sont susceptibles de provoquer la décharge de l'hormone posthypophysaire. C'est ainsi que CI.EVERLEY et FOLLEY (I970) notent à cet égard le rôle positif de 1'approche du vacher et de tous les stimuli auditifs ou visuels associés à la routine de traite. Ces faits avaient déjà été signalés précédemment par PEETERS, STORMORKEN et VANSchoubrork (I960) pour l'efficacité des excitations visuelles accompagnant la tétée et plus récemment par BRAND-

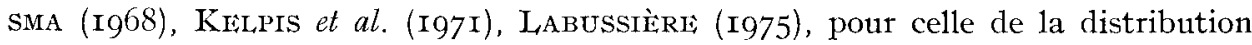
d'aliment concentré juste avant la traite.

L'intervention d'un réflexe conditionné permettant la libération d'ocytocine est l'hypothèse qui est généralement retenue pour expliquer l'effet bénéfique de ces différents facteurs d'ambiance. CLEVERLIEY et FOLLEY (I970) prétendent que ce 
réflexe peut s'installer après ro jours setılement d'apprentissage, mais l'École soviétique (ZAKS, I962) estime que le conditionnement est relativement difficile à établir puisqu'il nécessite parfois plus de roo répétitions (DyusEmbin, I963).

3. D'autres stimulations à point de départ mammaire, comme par exemple, la pose des gobelets sur les trayons peuvent provoquer le réflexe d'éjection ; FOLLEY et KNAGGS (I966) considèrent même qu'il s'agit là de la principale cause de la décharge d'ocytocine.

Ce fait avait déjà été pressenti par WhitTLESTONE (I946) ; selon cet auteur l'absence complète de pulsation ne permet pas d'obtenir le lait alvéolaire (si aucun massage préalable n'a été pratiqué) alors que 1'arrêt du pulsateur une minute après le branchement de la griffe ne modifie pas les conditions de la traite.

De nombreux chercheurs ont également souligné l'intérêt de machines à traire permettant de se dispenser de la préparation de la mamelle lorsque la pulsation est renforcée par l'air comprimé (BARTMANN, I966; TrogER et LOHR, I967 ; TeICHMANN et RUPPERT, I967), ou par tout autre système de gobelets à soufflets ou à piston qui stimulent rythmiquement la base du trayon (HAUPTMANN et SVARCBECK, I956; Abrasov et Kirenkov, r97o).

Sans aller jusqu'à l'acquisition de systèmes aussi compliqués, l'éleveur a bien entendu tout intérêt à choisir un matériel dont les paramètres de fonctionnement (rapport et vitesse de pulsation, niveau de vide, forme et taille des manchons, etc.) facilitent l'obtention dtu maximum de lait dans le minimum de temps (LABUsSIÈrE et RicharD, I965).

Il est plausible que dans notre expérience, la décharge d'ocytocine ait été provoquée par la pose des gobelets. Comme il n'existe aucune attente entre cette décharge et l'extraction du lait, nous sommes en mesure ainsi, de profiter pleinement de l'action bénéfique de cette hormone dont la durée de vie est courte ("période » inférieure à 2 minutes). Ceci pourrait expliquer que nous trouvions un débit maximum nettement plus élevé chez les animaux ne recevant aucune préparation et pourrait également justifier pourquoi nous n'observons pas d'allongement de la durée d'écoulement du lait à la suite de cette suppression.

Toutefois, les travaux de This,, Clough et Akam (1964) sur les fluctuations de vide sous le trayon et sur les perturbations du mouvement du manchon trayeur qui en résultent, suggèrent à quel point il pourrait être hasardeux de ne plus effectuer la préparation de la mamelle chez les vaches qui seraient traites avec une machine à traire mal installée, mal entretenue et déréglée. L'éleveur qui désirerait adopter cette méthode de travail simplifiée devra donc veiller tout particulièrement à ces derniers points mais il devra également tenir compte des répercussions possibles sur la qualité bactériologique de son lait.

En France, la qualité bactériologique du lait est appréciée par un dénombrement de la flore totale ou par une épreuve de réduction, selon que le lait est refroidi ou non. Notre législation est relativement peu exigeante à cet égard puisqu'on admet que le lait est de bonne qualité bactériologique lorsqu'il ne contient pas plus de 100 ooo microorganismes/m1. Les résultats présentés montrent que, sans lavage des mamelles, on peut non seulement satisfaire cette norme (même si dans le troupeau on trouve une certaine proportion de mamelles visiblement sales), mais pent-être aussi celle plus sévère recommandée en 1974 par la Fédération internationale de Laiterie : moins de 10 ooo micro-organismes $/ \mathrm{ml}$. Il ne faut pas oublier cependant que la contamination 
microbienne du lait par les mamelles non lavées s'ajoute à celle qui provient du matériel de traite ce qui peut aboutir à un dépassement des normes prescrites.

Sur un plan qualitatif, la flore microbienne dominante est composée de microorganismes que 1'on retrouve habituellement sur la mamelle (microcoques et corynébactéries), sur le sol ou dans la litière (corynébactéries, actynomycétales). Ces microorganismes ne se multiplient pas très facilement dans le lait et ne figurent pas parmi les agents habituels de sa dégradation. Le nombre de ces micro-organismes que l'on trouverait dans le lait en l'absence de lavage des mamelles ne paraît donc pas susceptible d'altérer la qualité bactériologique de celui-ci.

Par contre, la peau de la mamelle est une source importante de contamination par les spores de Bacillus (RIDGWAY, I958 ; LABOTS $c t$ al., I965 ; TERPLAN et WiESNER, I97I ; UNDERWOOD et al., I974) et par les spores de Clostridium du type butyrique (BERGÈRE et al., I968). Nos résultats montrent bien que le nombre de spores de Bacillus dans le lait provenant de mamelles non lavées est toujours nettement plus élevé que dans celui recueilli sur des mamelles nettoyées et UNDERWoOD ct al. (I974) signalent même qu'en hiver le nombre de spores de Bacillus aérobies mésophiles peut alors atteindre plusieurs centaines à plusieurs milliers par millilitre. Ceci peut être gênant pour l'industrie laitière car ces micro-organismes et particulièrement $B$. cerens limitent la durée de conservation du lait pasteurisé ou du lait UHT lorsqu'ils sont conditionnés aseptiquement (GROSSKOPF et HARPER, I969; LANGEFELD et al., I973; Mourgues et AUCLAIR, I973; Martin, 1974; OVErCAst et ATMARAN, I974).

Les inconvénients présentés par la présence dans le lait de spores de Clostridium fermentant le lactate sont bien connus des fromagers. Le risque de contamination est particulièrement élevé lorsque les vaches consomment de l'ensilage de qualité imparfaite. L'élimination de la moindre trace de bouse présente sur les mamelles est alors impérative si le lait produit est destiné à la fabrication de fromages tels que le Gruyère, 1'Emmenta1, 1'Edam, le Cheddar...

Enfin, sur un plan réglementaire, il ne faut pas oublier que le lait doit être propre $(J . O ., \mathrm{r} 97 \mathrm{I})$. Certes, la filtration à la ferme peut éliminer les impuretés grossières (mouches, poils, particules de paille ou de fourrage) mais pas les particules fines ou les matières solubles provenant des manelles souillées par l'urine ou la bouse et qui peuvent affecter les qualités organoleptiques du lait. La filtration n'est done pas une mesure palliant l'insuffisance de propreté de la traite.

Signalons enfin que, le lavage des pis est une des conditions d'attribution de la patente sanitaire (décret du I9 mars I963) et il figure régulièrement dans les propositions de règlements de la Communauté Économique Iuropéenne (1974). Il est également indispensable pour éliminer les résidus de produits de trempage des trayons souvent utilisés dans le cadre de la prophylaxie des mammites.

\section{V. - CONCLUSION}

Il ressort donc de ce travail que la suppression de la préparation de la mamelle n'entraîne pas de pertes importantes de production laitière. Elle se traduit par une simplification considérable de l'organisation du travail de traite puisque associée à la 
suppression de l'égouttage, un vacher peut alors traire seul entre 80 et roo vaches à l'heure dans une salle de traite en épi $2 \times 6$ (I2 postes) ne disposant d'aucun automatisme supplémentaire (LABUSST⿱亠ेRE, I975).

Sur le plan de la qualité bactériologique du lait, cette suppression peut être envisagée lorsque les mamelles sont propres (absence de bouse ou de produit de trempage des trayons) et dans les cas seulement où la présence dans le lait de spores de Bacillus ou de Clostridium ne pose pas de problèmes technologiques particuliers.

Cela impose de tout mettre en ceuvre, à la ferme, pour que les animaux soient propres en permanence (choix approprié de bâtiments, nettoyage des aires de repos et d'attente...) et que la vaisselle laitière soit correctement désinfectée.

Le lavage des mamelles occasionnellement sales ne devrait pas diminuer de façon appréciable 1'efficacité horaire du trayeur.

Reçu pour publication en avril 1976.

\section{SUMMARY}

SUPPRESSION OF PRE-MILKING UDDER MASSAGING AND WASHING IN DAIRY COWS. EFFECTS ON MILKING CHARACTERISTICS AND BACTERIOLOGICAL QUALITY OF THE MILK

I. Thirty three French Friesian black-and-white heifers were alternately distributed into 2 groups immediately after calving :

a) control group (I6 cows), massaging of the udder for 30 sec. with a wet towel previously soaked with hot water $\left(45^{\circ} \mathrm{C}\right)$. The attachment of the teat-cups took place immediately after cleaning of the udder ;

b) experimental group ( $\mathbf{I}$ cows), total suppression of udder stimulation from calving till drying-off.

2. Results obtained in the experimental group :

a) the 300 days-production of milk decreased by $2.31 \mathrm{p}$. 100 , whereas that of fat and crude protein increased by 5.82 and $2.6 \mathrm{I}$ p. Ioo, respectively. None of these 3 differences were significant. Conversely, the daily butterfat and protein levels were statistically higher (table I and fig. 2) and the milk volume collected at stripping $100 \mathrm{ml}$ larger, on an average, per milking (fig. 3 b) ;

b) although the time interval between attachment of the teat-cups and arrival of the alvcolar milk is always longer when no cleaning is made (fig. $3 c$ ), no lengthening of the milk flow time was recorded (fig. $3 \mathrm{e}$ ). This probably resulted from a substantially higher peak-flow at that moment (fig. $3 f$ ) ;

c) although remaining below the limits generally considered as indicators of a subclinical mammitis, the CMT notation was higher in cows not subjected to any preparation (fig. 4);

d) the microbial contamination of milk from the unwashed udders remained, on an average, lower than the 100 ooo total germs/ml corresponding to the standard value admitted by the French legislation for A-quality milk (table 2). If the udder is clean at the moment of milking, for instance because of adequate conditions of management, the contamination of the milk resulting from the absence of washing can be neglected.

\section{RÉFÉRENCES BIBLIOGRAPHIQUES}

AbBasov S. G., Kurenkov L. I., I970. Study under farm conditions of the "Volga " milking machine with new daw and nechanical milk ejection stimulations. Nauchno-tekh. Byull. Elektrif. Sel'sk Khoz(2) 29-34 (in Dairy Sci, Abstr., 34, I027). 
Barowicz T., Styczynski H., I974. Oxytocic activity measurements in blood plasma of cows during milking ejection (en polonais). Endokrynologia Polska, 25 (2), 83-93.

Bartmane R., I966. Comparison of mechanical and hand udder stimulation. Int. Z. Landw. (5), 526-530 (in Dairy Sci. Abstr., 29, 2 r93).

Bergitre J. L., Gouet P., Hermier J., Mocquot G., I968. Clostridia of the butyric group in milk products. Annls. Inst. Pasteur Lille 19, 4 1-54.

Brandsma S., I968. Stimulation by the milking machine. Proc. Symposium on Machine Milking, II9r 29, H. S. Hall (N.I.R.D. Reading).

Bryant M. P., Burkey L. A., I956. The characteristics of lactate fermentating sporeforming anacrobes from silage. J. Bacteriol., 71, 43 .

C.E.E., I974. Propositions de règlement du conseil relatif à des conditions sanitaires et de police sanitaire auxquelles doit réponđre le lait entier cru en tant que matière première pour la préparation de lait traité thermiquement et de ses dérivés R/I582/74 (AGRI 4II), Bruxelles.

Cleverley J. D., Folley S. J., 197o. The blood levels of oxytocin during machine milking in cows with some observations on its half-life in the circulation. $J$. Endocr., 46, 347-36r.

C.N.E.R.N.A., I962. Techniques de détection et de dénombrement des micro-organismes du lait. Cahiers Techniques du C.N.E.R.N.A., VII, p. 23.

Cousins C. M., I972. Sources of bacteria in farm bulk tank milk. J. Soc. Dairy Technol., 25, $200-204$.

Denamur R., I965. The hypothalamo-neurohypophysical system and the milk ejection reflex. Dairy Sci. Abstr., 2\%, 193-224 et 262-280.

DyUSEMBIN K. H., I963. Milking without suckling. Konevodstvo i. Konnye Sport., 33 (Io), Io (in Dairy Sci. Abstr., 2\%, I666).

Ely F., Petersen W. F., I94I. Factors involved in the ejection of milk. J. Dairy Sci., 24, 21 I-223.

F.I.L., I974. Qualité bactériologique du lait refroidi en vrac. Doc. 83 , bulletin annuel.

FitzPATRick R. J., ig6r. The estimation of small amounts of oxytocin in blood. In Oxytocin, 358-379, R. Caldeyro-Barcia et H. Heller, ed., Oxford Pergamon Press.

Folley S. J., Knaggs G. S., rg66. Milk ejection activity (oxytocin) in the external jugular vein blood of the cow, goat and sow in relation to the stimulus of milking or suckling. J. Endoct., 34, I97-2I4.

Ginsburg M., Smith M. W., I959. The fate of oxytocin in male and female rats. Br. J. Pharmac. Chomother., 14, 327-333.

Grifrin T. K., r968. General Discussion. Section III. Proc. Symposium on machine milking, I35-136, H. S. Hall N.I.R.D., ed., Reading.

Grosskopf J. C., Harper W. J., I969. Role of psychophilic sporeformers in long life milk. J. Dairy Sci., $52(6), 897$.

Hauptmann J., Svarcbeck J., I956. New method of functioning of milking machines. I et II. Sborn. Cesk. Akad. Zemed. Ved., 29, $211-22,353-366$.

IPSEN E. J., I964. Milking and byre conditions of 5000 milk-rccorded herds with special reference to the effect of these conditions on level of production. Tidsskr Landokon (4), 247-267 (in Dairy Sci. Abstr., 29, 3368).

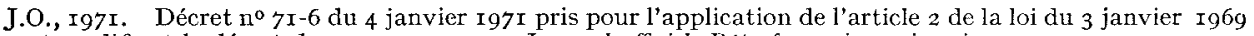
et modifiant le décret du 25 mars 1924. Journal officiel, Rép. française, 7 janvier I971, p. 270.

Johns C. K., I962. The coliform counts of raw milk as an index of udder cleanliness. Congr. Int. Lait. Copenhague, C, 365-37I.

Kelpis E. A., Lusis M. E., Mezhale Z. A., Serzhane M. K., Laurs A. R., i97i. Study of stimulation of milk ejection in automated milking installations. Trudy latviisk. Sel'Skokhoz Akad, 34, 23-30 (in Dairy Sci. Abstr., 33, 5203).

Knoop C. E., Monroe C. F., I950. Influence of pre-milking preparation of cow's udder upon the let down of milk. J. Dairy Sci, 33, 621-632.

Labots H., Hup G., Galesloot Th. E., 1965. Bacillus ceveus in raw and pasteurized milk. III. The contamination of raw milk with $B$. cereus spores during its production. Neth. Milk Dairy J., 19 (3), I9I-22I.

Labussière J., Richard Ph., I965. La traite mécanique. Aspects anatomiques, physiologiques et technologiques. Mise au point bibliographique. Ann. Zootech., 14, 63-1 26.

Labussière J., Martinet J., ig64. Description de deux appareils permettant le contrôle automatique des débits de lait au cours de la traite à la machine. Premiers résultats obtenus chez la brebis. Ann. Zootech., 13, I99-212.

LAbussì̀re J., I970. Comment peut-on concevoir la traite des vaches au cours des années à venir. Revue de l'Elevage (10), 25-37.

Labussière J., I972. Comment améliorer l'efficacité du trayeur. In L'exploitation moderne du troupeau laitier. Numéro hors-série de l'Élevage, 177-1 88.

LAbussik̀re J., I975. Limitation des servitudes de la traite. Choix des méthodes et des équipements. Conduite de l'exploitation laitière en période d'accroissement des charges de production. Journées d'étude de l'I.T.E.B., I4-I5 janvicr 1975. I.T.E.B., I47-I 49 rue de Bercy 75579 Paris cedex I2.

Langefeld L. P. M., Cuperus F., Stadhouders J., I973. Bactériological aspects of the keeping quality at $5^{\circ} \mathrm{C}$ of reinierted and non reinferted pasteurized milk. Neth. Milk Dairy J., 27, 54-65. 
Martin J. H., 1974. Significance of bacterial spores in milk. J. Milk. Food. Technol., 27, 94-98.

Momongan V. G., Schmidt G. H., I970. Oxytocin levels in the plasma of Holstein-Friesian Cows during milking with and without a premilking stimulus. J. Dairy Sci., 53 (6), 747-75 I.

Morag M., Saran-Rosenzuaig A., Sagi R., 1973. The effect of pre-milking udder stimulation on milk yield in Israeli-Friesian cows (communication personnelle).

Mourgues R., Auclair J., I973. Durée de conservation à $4^{\circ} \mathrm{C}$ et $8^{\circ} \mathrm{C}$ du lait pasteurisé conditionné aseptiquement. Lait $L I I I, 528,48 \mathrm{I}-490$.

Murray A. J. W., Lorna G. Lightrody, 1962. Fffect of different pre-milking methods on the milking characteristics of "Australian Illawara Shorthorn cows". Od J. Agric. Sci., 19, 255-265.

Overcast W. W., Atmaram K., 1974. The role of Bacillus cereus in sweet curdling of fluid milk. J. Milk Food Technol., 37, 233-236.

Peeters G., Stromorken H., Vanschoubroek F., rg6o. The effect of different stimuli on milk ejection and diuresis in the lactating cow. J. Endocr., 20, I63-172.

Phillips D. S. M., I960. The effect of pre-milking stimulation on milk and butterfat production. Proc. N. Z. Soc. Anim. Prod., 20, 93-94.

Phillips D. S. M., I965. Is pre-milking stimulation worth while? Proc. Ruak. Farm. Conf. Week, 223-235.

Phillips D. S. M., Cowhig M. J., Walshe M. J., I967. Dairy Husbandry department pre-milking stimulation. Anim. Husb. Fairying div. Anim. Sc. Dir., 6r-62.

RidGway J. D., I958. The incidence and thermal resistance of mesophilie spores found in milk and related environnements. J. Appl. Bact., 21, I18-127.

Schnfider C., I954. Traité pratique des essais du lait. I I éd., 50-51. Les Hoirs C. J. Wiss S. A., Berne.

Teichmann G., Ruppert P., I973. Use of the Physiomatik milking system on a commercial dairy farm. (en allemand). Tierzucht, 27 (12), 557-562 (in Dairy Sci. Abst., 36, 3336).

Terplan G., Wiesner H. U., I97I. Evaluation of cowshed with regard to milk production hygiene. Arch. Lebensmittelhyg., 22, $15^{8-159 .}$

Thiel C. C., Clougr P. A., Akam D. H., I964. Mechanics of machine milking ${ }^{\circ}{ }^{\circ}$ Pressure in the teatcup assembly and liner wall movement. J. Dairy Sci., 31, 303-314.

Troger F., LoHr H., I967. Verfahron zur Mechanisierung des Anrüstens (Euter massage) beim melken der Kühe mit der Melkmaschine. Tierzucht, 21 (4), I84-188.

Unde Rwood H. M., McKinnon C. H., Davies F. L., Cousins C. M., I974. Sources of Bacillus spores in raw milk. $X I X^{\mathbf{e}}$ congr. Intern. Lait., B 6, 406-407.

Whittlestone W. G., I946. The effect of the level of stimulus applied by the pulsation on the rate of machine milking. N. Z. J. Sci. Tech., 27, A 445 .

Whittlestone W. G., Parkinson R. D., McFetridg E. M. J., 1957. More milk from good milking methods. N. Z. Dairy Exp., 33, 58-62.

WIIson G. G., 1963. Some aspects of machine milking rate. J. Dairy Res., 30, 191-196.

ZAKs M. G., I962. In The motor apparatus of the mammary gland. Ed. A. ' $\mathrm{T}$. Cowie : Oliver and Boyd, Edinburgh and London. 